\title{
Transparency of magnetosphere for cosmic rays in last two millennia
}

\section{Daniel Gecášek, ${ }^{a, *}$ Pavol Bobík ${ }^{b}$ and Ján Genči ${ }^{a}$}

${ }^{a}$ Technical University of Košice, Department of computers and informatics, Letná 9, Košice, Slovakia

${ }^{b}$ Institute of Experimental Physics, Slovak academy of sciences, Watsonova 47, Košice, Slovakia

E-mail: daniel.gecasek@tuke.sk, bobik@saske.sk, jan.genci@tuke.sk

We present a simulation of magnetosphere transparency for cosmic rays in the last two millennia. Simulations were done in the COR system, in a module for cosmic rays trajectory evaluation in different models of geomagnetic fields for the period 0 to $1900 \mathrm{CE}$. The COR system available at cor.crmodels.org is also briefly presented. The global and local trends for cut-off rigidities and cosmic rays intensity evolution in the last two millennia are analyzed.

$37^{\text {th }}$ International Cosmic Ray Conference (ICRC 2021)

July 12th - 23rd, 2021

Online - Berlin, Germany

\footnotetext{
${ }^{*}$ Presenter
} 


\section{Introduction}

The magnetosphere has changed significantly in the last millennia. Consequently, long-term changes in the transparency of the magnetosphere for cosmic rays could have an impact on climate studies and on cosmogenic radionuclides production [1], and therefore on dating analysis methods results and precision.

For example, in their article [2], Shea and Smart suggest that the long-term change in the cosmic ray $(\mathrm{CR})$ intensities at the top of the atmosphere in the last four centuries at specific locations around the globe should be considered in studies of possible relationships between cosmic radiation and the Earth's climate.

In this article, we present a simulation of magnetosphere transparency for CRs in the last two millennia as an example of a result from the CutOff Rigidity (COR) system. The COR system is dedicated to the evaluation of CR trajectories in Earth's magnetosphere. Note that, when we mention CRs in this article, we mean protons. The COR system will be briefly introduced in the next section of this article.

\section{CutOff Rigidity system}

The COR system, which is available at https: //cor . crmodels . org/, offers various models and input modes for CR trajectory simulation in the Earth's magnetosphere, it also offers selected models of the Earth's magnetosphere. The input modes of CR trajectories simulation in geomagnetic field are as follows:

- Vertical: Vertical direction of incoming CR trajectories;

- Multidirectional: Multiple directions uniformly covering all incoming directions from a $2 \pi$ solid angle;

- Space-time point grid: A grid of points in space and time for either vertical or multidirectional mode;

- Individual/single trajectory visualisation.

Two simulation modes are available for vertical and multidirectional input modes, as follows:

- Normal: All rigidities from the start of the interval to $100 \mathrm{GV}$ with rigidity step given as input are evaluated. The start of the interval is the same as the rigidity step (usual options are $0.01 \mathrm{GV}$ and $0.1 \mathrm{GV})$;

- First allowed trajectory: The simulation stops when the first of the evaluated trajectories is allowed. Because the order of rigidity values simulated is given, the first allowed trajectory will be one with the lowest cut-off rigidity. Although simulations in this mode are guaranteed to be faster than in the normal mode, the result is limited to the lowest cut-off rigidity.

All of the modes of the simulation include an evaluation of the internal geomagnetic field intensity, which is computed from the IGRF model and external geomagnetic field intensity computed from either T05 or T96 models [3]. 
A space-time point grid input mode is available for magnetic field model simulation. The magnetic field models that are currently available are combinations of external field models T96, T05, and internal field model IGRF. In the future, we plan to add newer Tsyganenko models into the system. In the following subsections, we will present some chosen visualisations that showcase the capabilities of our system.

\subsection{Cut-off rigidity sky map}

In figure 1 we see a sky map of cut-off rigidities. The three figures display maps for lower, upper and effective cut-off rigidities respectively. The sky map was evaluated from cut-off rigidities for 576 incoming directions uniformly covering a $2 \pi$ sphere above the selected position. The example shows a sky map for the Lomnický štít observatory for 10th of June 1998, which was evaluated with the selected model of external geomagnetic field T05 and internal geomagnetic field IGRF model.

Note the East-West asymmetry, where we have lower cut-off rigidities on the West. The map shows that vertical effective cut-off rigidity here is approximately $3.5 \mathrm{GV}$, and vertical cut-off rigidities of trajectories from the West direction over the horizon reach $2.7 \mathrm{GV}$. Trajectories coming from the East direction over the horizon have effective cut-off rigidities that rapidly rise until 30GV. Precise values of effective cut-off rigidities for all 576 directions can be found in the cut-off rigidity catalog.
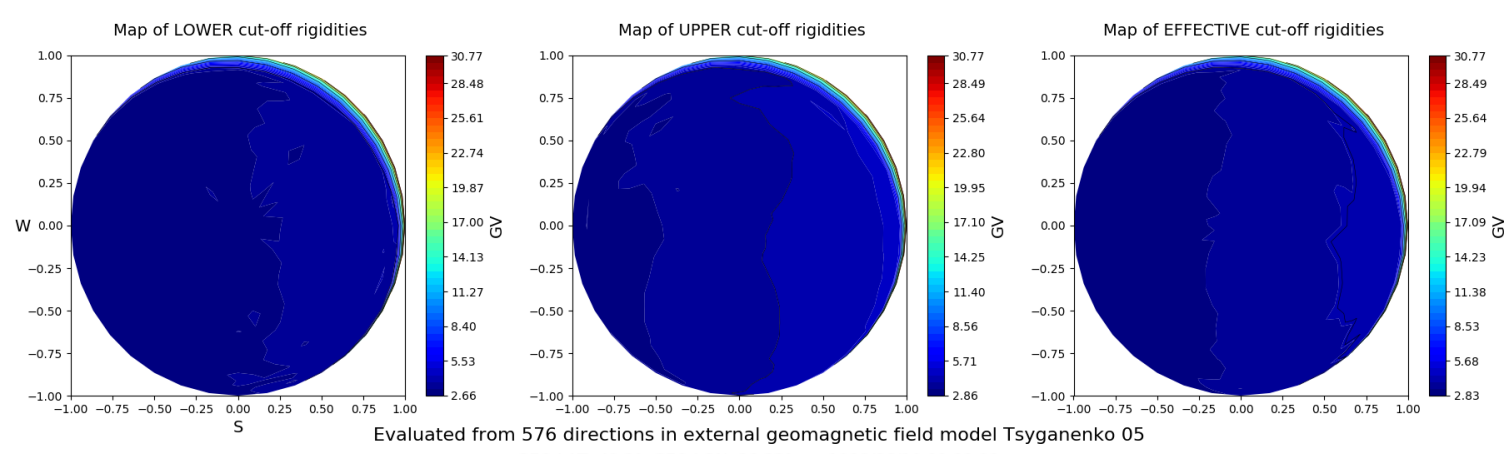
$\begin{array}{ll}576 & \text { directions in external geomagnetic field model } \\ \text { GEO LAT: } 49.2^{\circ} \text { GEO LON: } 20.22^{\circ} & 1998 / 06 / 1000: 00: 00\end{array}$

Figure 1: Cut-off rigidity sky map (for more details, see the text).

\subsection{Cut-off rigidity spectra catalog}

Figure 2 shows a page from a catalog of spectra of allowed and forbidden cut-off rigidities. The whole catalog consists of 577 pages. In the right-hand panel, the evaluated directions of incoming trajectories are displayed as black points. A specific direction, in which the spectrum of allowed and forbidden cut-off rigidity is displayed on the left-hand side, and is marked as a red point. In this specific case, the displayed cut-off rigidity is a trajectory that comes from the vertical direction.

In the left-hand panel, there is a spectrum of allowed and forbidden rigidities with information about the geographic position for which the trajectories were simulated at the specified time. The lower, upper and effective rigidities are highlighted with arrows and their values are explicitly stated under the diagram. The external model of the magnetic field that was used is also specified. 

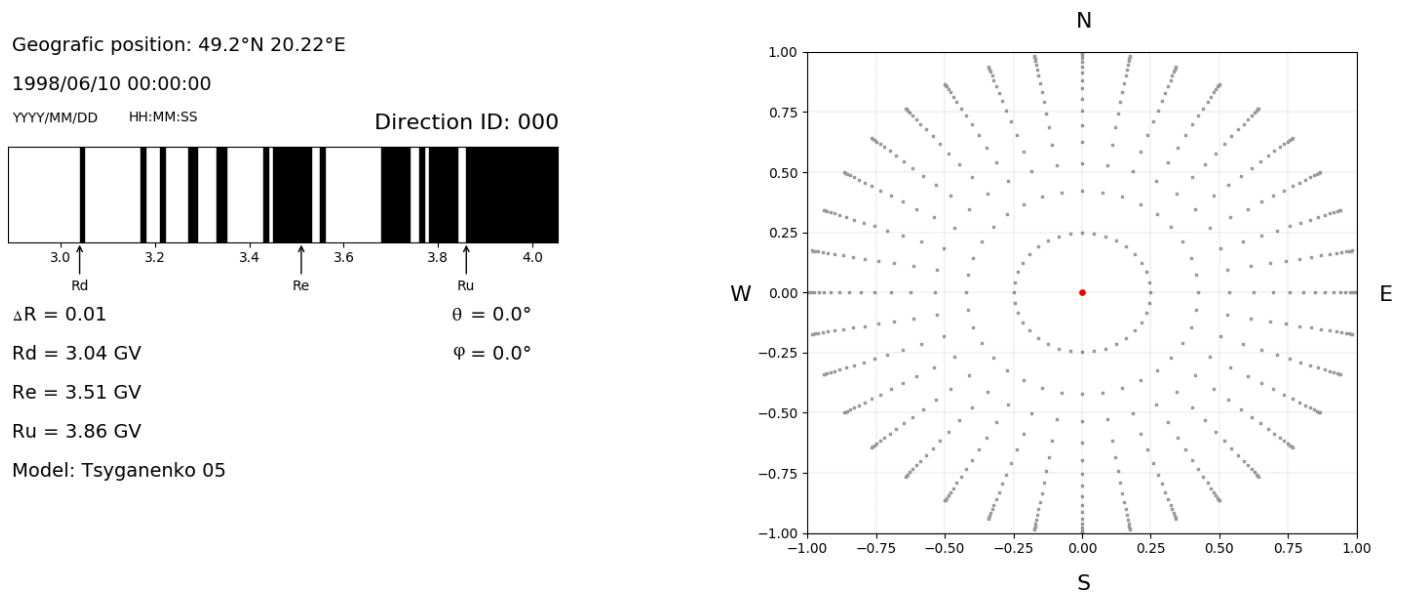

Figure 2: A page of a catalog of cut-off rigidity spectra (for more details, see the text).

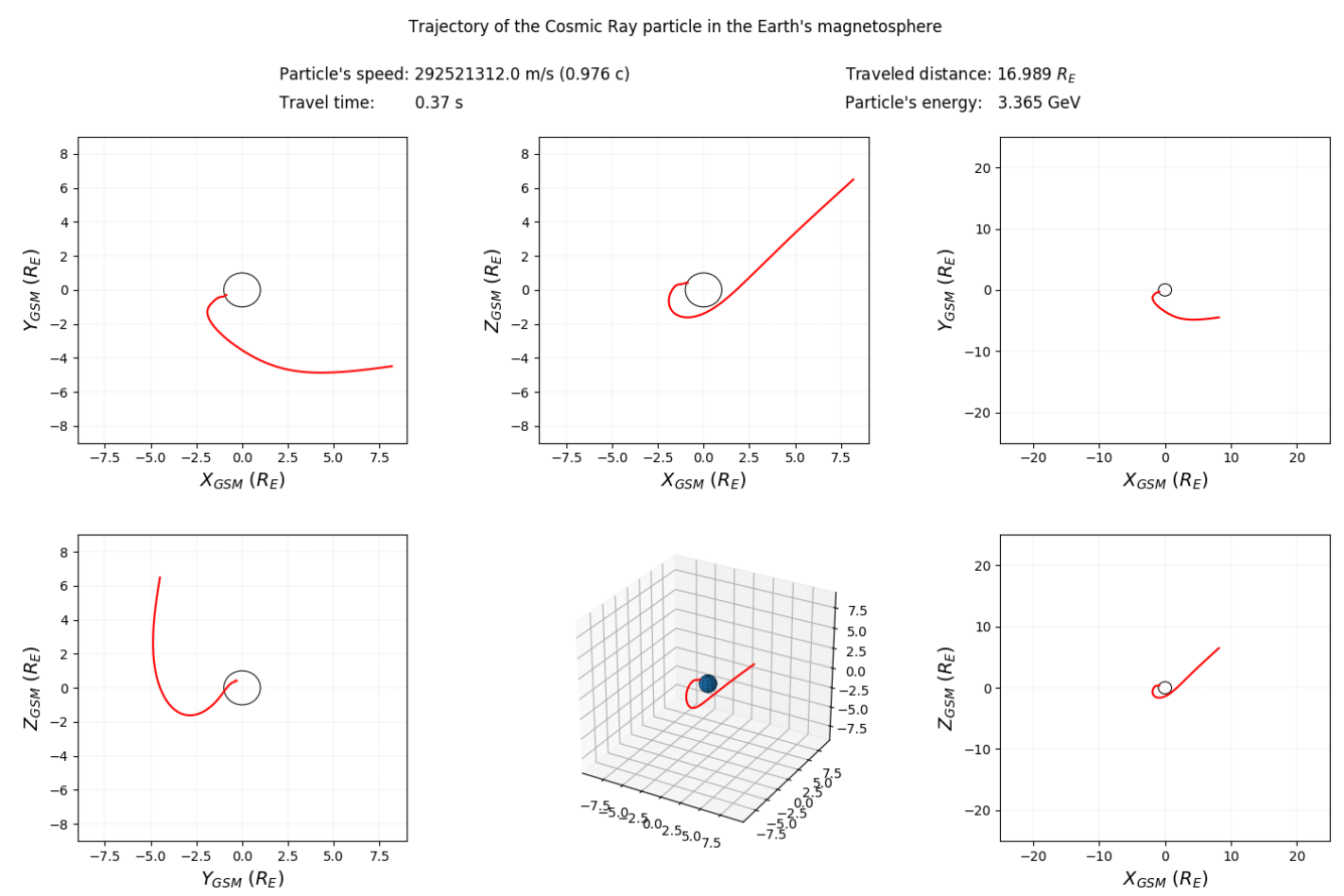

Figure 3: Visualisation of a specific trajectory (for more details, see the text).

\subsection{Visualisation of a single trajectory}

The visualisation that is presented in figure 3 shows the trajectory of a CR particle, specifically a proton with energy $3.365 \mathrm{GeV}$ or $4.2 \mathrm{GV}$ rigidity. The trajectory is shown as a red line, in five different 2D projections in the GSM coordinate system and from a 3D perspective.

\section{Methods to evaluate the transparency of the magnetosphere}

We evaluated vertical cut-off rigidities over the whole globe with $10^{\circ}$ in latitude and $5^{\circ}$ in longitude steps for a period from 0 to 1900CE. The set of geomagnetic field models that is 


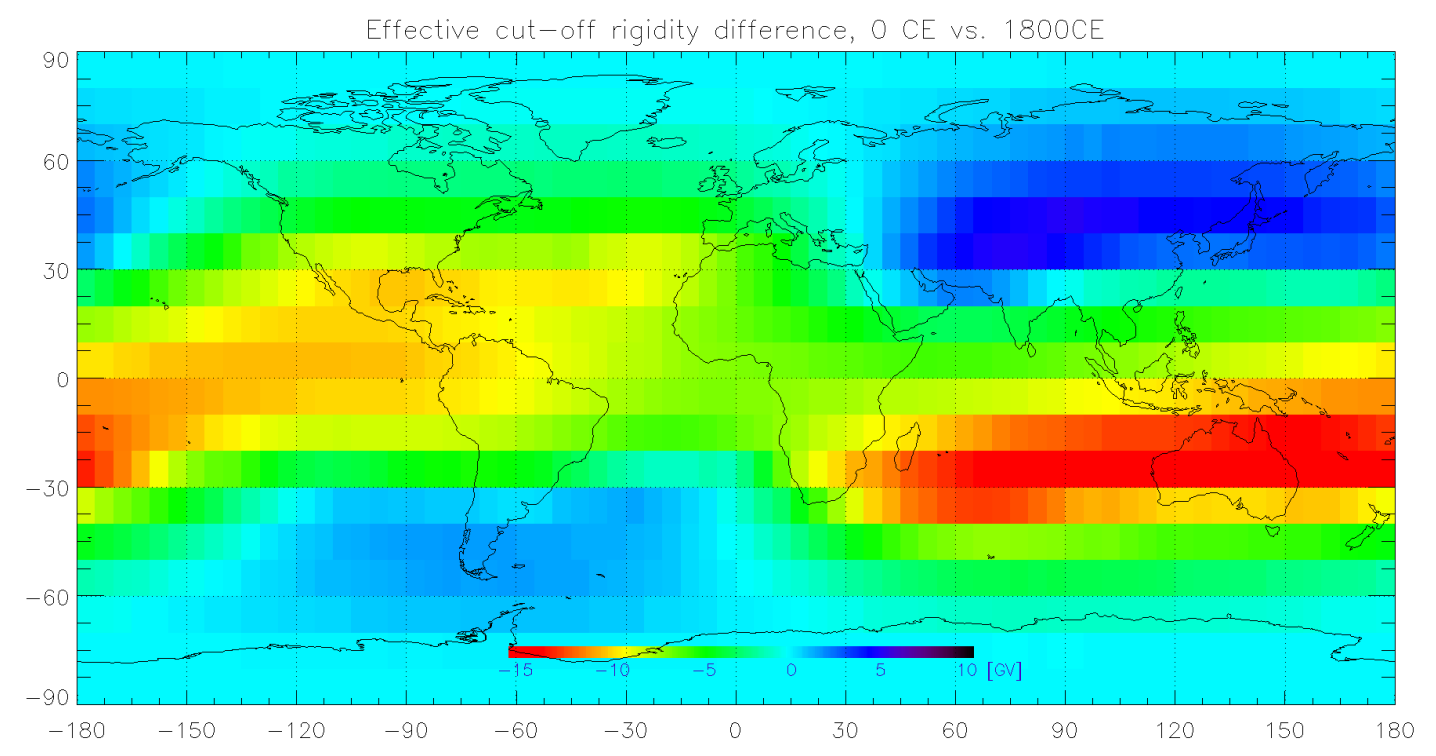

Figure 4: Differences of effective cut-off rigidities in the last two millennia (for more details, see the text).

presented in [4] was used. We used backtracing method to evaluate the trajectories of CRs in the magnetosphere, which is described in detail in [4][5].

The evolution of cut-off rigidities in the last two millennia leads to the differences that are shown in Figure 4. The difference map between cut-off rigidities in the year 0 and 1800CE shows that rigidities decrease at the bigger part of the Earth's surface. However, there are regions where the situation is opposite and rigidities increase. In those regions where rigidities increased, the values of increase are smaller than the decreasing values in regions with the opposite pattern.

The geomagnetic cut-off rigidities in the last two millennia changes significantly in some regions, as shown in Table 1. This table presents some examples of positions on the Earth with notable changes in vertical cut-off rigidity. For example, the vertical cut-off rigidities changes from $21 \mathrm{GV}$ two thousand years ago to $6 \mathrm{GV}$ in the year 1800 in Southern Australia (i.e., the absolute change there is around $15 \mathrm{GV}$ ). Northern Australia and the Indian Ocean are regions with the highest values of absolute change in rigidity. The effective cut-off rigidity in the Cairo area, whose position is noted in Table 1 . only has a $0.62 \mathrm{GV}$ change between the years 0 and $1800 \mathrm{CE}$, and represents a position with a small change. Regions with middle latitudes in Asia record an increase in cut-off rigidities values that are close to $5 \mathrm{GV}$.

\subsection{Cosmic ray intensity at the top of the atmosphere in the last two thousand years}

In the following step, we evaluated the spectra of CR protons at the top of the atmosphere for all positions and periods. They were evaluated from spectra of allowed and forbidden rigidities, and from proton spectra at the magnetosphere border (i.e., the magnetopause).

In the model, we apply two limit spectra as a spectrum at the magnetopause (i.e., low and high-intensity estimation of the spectrum at $1 \mathrm{AU})$. We select these limit spectra from force-field approximation spectra, with the $\phi$ published in Table 1 of article [8], covering the last millennium. The table for the Maunder minimum shows value of $\phi=300 \pm 50 M V$ and Spörer minimum 
Table 1: Selected regions with significant changes in effective cut-off rigidities between years 0 and $1800 \mathrm{CE}$.

\begin{tabular}{cccccc}
\hline Latitude & $\begin{array}{c}\text { Longitude } \\
R_{e} \text { in } 0 \\
{[\mathrm{GV}]}\end{array}$ & $\begin{array}{c}R_{e} \text { in 1800CE } \\
{[\mathrm{GV}]}\end{array}$ & $\begin{array}{c}\text { Difference } \\
{[\mathrm{GV}]}\end{array}$ & $\begin{array}{c}\text { Region } \\
\text { name }\end{array}$ \\
\hline$-30^{\circ}$ & $145^{\circ}$ & 20.99 & 5.99 & -15.00 & Southern Australia \\
$-30^{\circ}$ & $150^{\circ}$ & 21.21 & 6.13 & -15.08 & Southern Australia \\
$-30^{\circ}$ & $155^{\circ}$ & 21.38 & 6.38 & -15.00 & Southern Australia \\
$30^{\circ}$ & $270^{\circ}$ & 14.22 & 6.05 & -8.17 & New Orleans area \\
$20^{\circ}$ & $270^{\circ}$ & 18.26 & 10.38 & -7.88 & Yucatán \\
$30^{\circ}$ & $30^{\circ}$ & 10.80 & 10.18 & -0.62 & Egypt, Cairo area \\
$40^{\circ}$ & $120^{\circ}$ & 5.77 & 10.06 & 4.29 & Beijing area \\
\hline
\end{tabular}

$\phi=290 \pm 45 M V$. These are the lowest values of modulation potential in the last millennium and we use the spectrum for $\phi=290 M V$ as a maximum intensity spectrum. The Wolf-Spörer maximum in a cited table has $\phi=560 \pm 110 \mathrm{MV}$, which we took as a minimum intensity spectrum.

The spectrum at $1 \mathrm{AU}$ during the last two thousand years probably has extreme values with higher and lower intensities than the limit spectra that we used. To estimate them, we took the minimum extreme spectrum from the force-field approximation spectra for the period 1951-2016 that were published in [6]. As a minimum extreme intensity spectrum, we took the spectrum from June 1991 with $\phi=1360 M V$. The period from 1951 to 2016 includes a deep solar minimum that is close to the year 2009, where the measured monthly spectrum from September 2009 with maximum recorded intensity has force-field approximation with $\phi=389 M V$. This value is higher than the modulation potential for the Spörer and Maunder minimum, thus we did not set a new maximum extreme spectrum.

The validity of approximation of the limit spectra based on the last millennium for the last two millennia could be supported by solar sunspot numbers, as reconstructed from $14 \mathrm{C}$ measurements in article [7]. This shows the highest activity during the period between 0 and 1900CE in the nineteenth century. Thus, an estimation of the minimum intensity spectrum that is based on the Wolf-Spörer maximum with a higher modulation potential than the Dalton and Maunder-Dalton maxima is probably a good place to start an approximation.

The results from the simulations shown in Tables 2 and 3 indicate that changes in CR intensities from the magnetosphere field evolution in the last two thousand years was remarkably stronger than changes from the Sun's activity. Table 2 shows changes of intensities for two limit spectra, for minimum intensity spectrum with $\phi=560 M V$ and maximum intensity spectrum with $\phi=290 M V$. As table 3 with extreme minimum intensity spectrum with $\phi=1360 M V$ shows, even if the variation in solar modulation is stronger than the used limit cases, this statement still remains valid. The relative difference between CR intensities in 0 and 1800CE is smaller for higher modulation potentials.

The map in Figure 5 shows changes in CR intensities at the top of the atmosphere between the years 0 and $1800 \mathrm{CE}$ for the assumed extreme minimum intensity spectrum. The regions where the intensity increased by more than $400 \%$ are indicated by red color. These regions include large parts of the Indian Ocean, Australia, and a significant part of New Zealand. The intensity increase is close to $200 \%$ over North America, and the north part of Atlantic and Spain. There are two regions 
Table 2: Selected regions with significant changes in CR intensities between years 0 and $1800 \mathrm{CE}$ for minimum and maximum limit spectra.

\begin{tabular}{cccccc}
\hline Latitude & Longitude & $\begin{array}{c}I \text { in } 0 \\
{\left[p /\left(\mathrm{m}^{2} . s . s r\right)\right]} \\
560 \mathrm{MV} / 290 \mathrm{MV}\end{array}$ & $\begin{array}{c}I \text { in } 1800 \mathrm{CE} \\
{\left[\mathrm{p} /\left(\mathrm{m}^{2} . s . s r\right)\right]} \\
560 \mathrm{MV} / 290 \mathrm{MV}\end{array}$ & $\begin{array}{c}\text { Relative difference } \\
\text { in [\%] } \\
560 \mathrm{MV} / 290 \mathrm{MV}\end{array}$ & $\begin{array}{c}\text { Region } \\
\text { name }\end{array}$ \\
\hline$-30^{\circ}$ & $145^{\circ}$ & $39 / 41$ & $328 / 374$ & $738 / 817$ & Southern Australia \\
$30^{\circ}$ & $270^{\circ}$ & $80 / 84$ & $322 / 367$ & $354 / 384$ & New Orleans area \\
$20^{\circ}$ & $270^{\circ}$ & $41 / 43$ & $139 / 151$ & $238 / 251$ & Yucatán \\
$40^{\circ}$ & $355^{\circ}$ & $200 / 220$ & $575 / 691$ & $188 / 214$ & Spain \\
$30^{\circ}$ & $30^{\circ}$ & $127 / 137$ & $145 / 157$ & $14 / 15$ & Egypt, Cairo area \\
$40^{\circ}$ & $90^{\circ}$ & $447 / 524$ & $149 / 162$ & $-67 /-69$ & North China \\
$-50^{\circ}$ & $290^{\circ}$ & $589 / 710$ & $364 / 418$ & $-38 /-41$ & South Argentina \\
\hline
\end{tabular}

Table 3: Selected regions with significant changes in CR intensities between years 0 and 1800CE for extreme minimum intensity spectrum.

\begin{tabular}{cccccc}
\hline Latitude & Longitude & $\begin{array}{c}I \text { in } 0 \\
{\left[p /\left(m^{2} . s . s r\right)\right]}\end{array}$ & $\begin{array}{c}I \text { in } 1800 \mathrm{CE} \\
{\left[p /\left(\mathrm{m}^{2} . s . s r\right)\right]}\end{array}$ & $\begin{array}{c}\text { Relative difference } \\
1360 \mathrm{MV}[\%]\end{array}$ & $\begin{array}{c}\text { Region } \\
\text { name }\end{array}$ \\
\hline$-30^{\circ}$ & $145^{\circ}$ & 35 & 231 & 564 & Southern Australia \\
$30^{\circ}$ & $270^{\circ}$ & 67 & 228 & 284 & New Orleans area \\
$20^{\circ}$ & $270^{\circ}$ & 37 & 111 & 204 & Yucatán \\
$40^{\circ}$ & $355^{\circ}$ & 152 & 357 & 134 & Spain \\
$30^{\circ}$ & $30^{\circ}$ & 102 & 115 & 12 & Egypt, Cairo area \\
$40^{\circ}$ & $90^{\circ}$ & 294 & 118 & -60 & North China \\
$-50^{\circ}$ & $290^{\circ}$ & 364 & 251 & -31 & South Argentina \\
\hline
\end{tabular}

where intensity decreases: the largest region is over Siberia, North China, Korea, and Japan, where the intensity decreases by 50\%; and the other region is over the southern part of South America, where the decrease was around $30 \%$.

\section{Conclusions}

This paper has briefly presented the use of the COR system to evaluate CR trajectories in the Earth's magnetosphere. As an example of a result of the COR system, we present a simulation of magnetosphere transparency for CRs in the last two millennia. The preliminary results show that changes in CR intensities from magnetosphere field evolution in the last two thousand years were remarkably stronger than changes from the Sun's activity.

\section{Acknowledgment}

The PECS project TUKE Space Forum is acknowledged. PB acknowledges the Slovak VEGA grant agency, project 2/0077/20, for their support. 


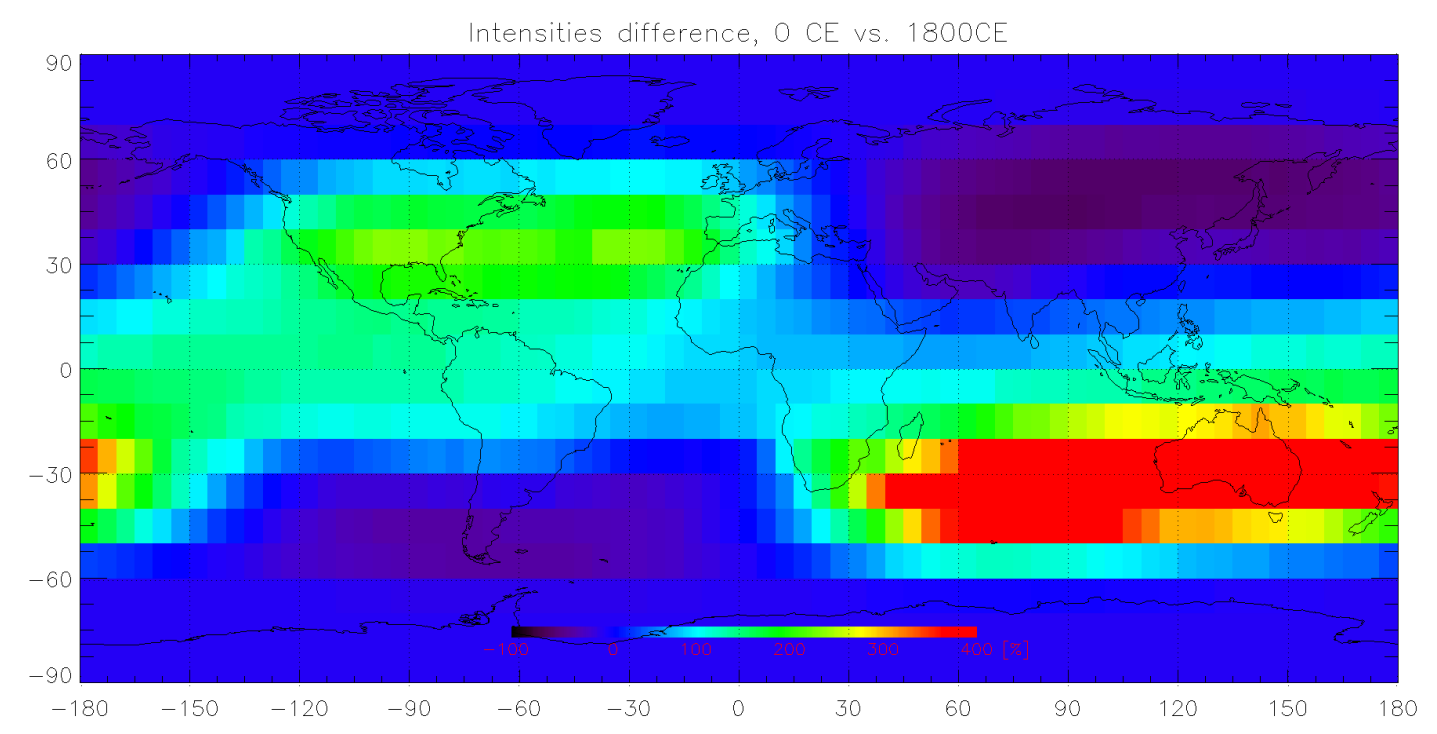

Figure 5: Change in CR intensities at the top of the atmosphere between the years 0 and 1800CE for extreme minimum intensity spectrum with $\phi=1360 M V$ (for more details, see the text).

\section{References}

[1] Kovaltsov, G.A., Mishev, A., and Usoskin, I.G. "A new model of cosmogenic production of radiocarbon ${ }^{14} \mathrm{C}$ in the atmosphere" Earth and Planetary Science Letters 337, 114, 2012

[2] Shea, M. A. and Smart, D. F. "Preliminary Study of the 400-Year Geomagnetic Cutoff Rigidity Changes, Cosmic Rays and Possible Climate Changes", International Cosmic Ray Conference, $2003,7,4205$

[3] Tsyganenko NA and Sitnov MI, "Modeling the dynamics of the inner magnetosphere during strong geomagnetic storms", Journal of Geophysical Research: Space Physics, 110, A3, 2005

[4] Kudela, K. and Bobik, P. "Long-Term Variations of Geomagnetic Rigidity Cutoffs" Solar Physics 224, 423, 2004

[5] Bobik et. al. "Magnetospheric transmission function approach to disentangle primary from secondary cosmic ray fluxes in the penumbra region" Journal of Geophysical Research (Space Physics) 111, A05205, 2006

[6] Usoskin, I.G., Gil, A., Kovaltsov, G.A., Mishev, A.L., and Mikhailov, V.V. "Heliospheric modulation of cosmic rays during the neutron monitor era: Calibration using PAMELA data for 2006-2010" Journal of Geophysical Research (Space Physics) 122, 3875, 2017

[7] Usoskin, I.G., Solanki, S.K. and Kovaltsov, G.A. "Grand minima and maxima of solar activity: new observational constraints”, Astron. Astrophys., 471, 301, , 2007

[8] Brehm N. et al. "Eleven-year solar cycles over the last millennium revealed by radiocarbon in tree rings", Nature Geoscience, 14, 1, 10, 2021 